\title{
Multilinguales
}

\section{Girolamo Merolla au Congo : récits de " colorisme » chez un missionnaire capucin de la fin XVII ${ }^{\mathrm{e}}$ siècle}

$$
\text { جيغولامو مغولا في الكونغو :قصص"لون العرق "عند راهب كبوشي في نهاية }
$$

José Sarzi Amade

\section{(2) OpenEdition}

\section{Journals}

Édition électronique

URL : http://journals.openedition.org/multilinguales/406

DOI : $10.4000 /$ multilinguales.406

ISSN : 2335-1853

Éditeur

Université Abderrahmane Mira - Bejaia

Référence électronique

José Sarzi Amade, « Girolamo Merolla au Congo : récits de « colorisme » chez un missionnaire capucin de la fin XvII e siècle », Multilinguales [En ligne], 8 | 2017, mis en ligne le 01 juin 2017, consulté le 23 septembre 2019. URL : http://journals.openedition.org/multilinguales/406 ; DOI : 10.4000/ multilinguales.406

Ce document a été généré automatiquement le 23 septembre 2019.

\section{(i) $\odot$

Multilinguales est mise à disposition selon les termes de la Licence Creative Commons Attribution Pas d'Utilisation Commerciale - Pas de Modification 4.0 International 


\title{
Girolamo Merolla au Congo : récits de « colorisme » chez un missionnaire capucin de la fin XVII siècle
}

\author{
جيغولامو مغولا في الكونغو :قصص"لون العرق "عند راهب كبوشي في نهاية \\ القرن الثامن عشو عشير
}

José Sarzi Amade

1 Le prêtre capucin Girolamo Merolla, originaire de Sorrente, laissa derrière lui une œuvre intitulée : Breve e Succinta Relatione del Viaggio nel Regno di Congo [... ${ }^{1}$, parue en 1692. De ce livre, peu de choses avaient été révélées, mais mon intervention exhaustive le concernant, a permis une réévaluation de son contenu ${ }^{2}$.

2 Nous sommes en présence d'un récit de voyage divisé en deux parties. La première partie de livre du Capucin narre les faits depuis son départ d'Italie, de Naples, jusqu'à sa prise de fonction dans la mission du comté de Soyo, appartenant au Royaume du Congo, à partir du 27 mai 1683, et les quelques actions personnelles qu'il accomplit dans ce comté. Cependant, nous trouvons une narration qui est décousue, conséquence de la piètre santé de Merolla. En effet, il sera presque continuellement alité dès 1685 . La seconde partie du livre met en scène un missionnaire dont la santé est en sursis : Merolla y narre ses péripéties, à partir de 1688 , pour tenter d'amener à la foi un royaume voisin du Congo, le Kakongo, et de couronner un roi afin de rétablir la souveraineté perdue du Congo. Ces deux actions diplomatiques se soldèrent par un échec en raison d'une rechute de Merolla dans la maladie et de rapports de forces néfastes d'un Royaume du Congo sombré dans l'anarchie, aux prises avec les différentes entités coloniales, c'est-à-dire le Portugal, la Hollande et l'Angleterre.

La langue de Breve e Succinta Relatione peut apparaître aujourd'hui comme ampoulée, heurtée et manquant de fluidité (sans doute même pour le lecteur de l'époque). En réalité, ce récit fut aménagé par le Père Angelo Piccardo da Napoli, figure de 
"descriptor ", qui retranscrit, vraisemblablement sous la dictée de Merolla, la narration qu'il écoutait, sans gommer ni altérer le caractère spontané de la langue orale. En qualité de scripteur, il est probable qu'il incorpora au texte, parfois à la hâte ou de façon inappropriée, ses propres références ou commentaires. Piccardo reconnaît d'ailleurs qu'il dut composer ce livre dans la précipitation et demande à son lecteur de ne pas lui en tenir rigueur:

Quantunque potrei pure con brevità aggiungerli cotesta Breve e succinta Relazione esser ancora figlia della Brevità del Tempo, caggionata dalla sollecitudine dell'Autore aspirante alla seconda partenza per quell'Africane Maremme, [...]. [7r $]^{4}$

La rapidité avec laquelle fut composé ce livre, chose peu courante pour les récits du même acabit, sera même soulignée par Piccardo qui avertit le lecteur du caractère inachevé voire bâclé de l'œuvre. Néanmoins, c'est lui qui lui donna un style historique, un ornement rhétorique ou une amplification épique, et donc qui héroïsa son compagnon Merolla.

5 En définitive, comme l'a fort bien expliqué Sylvie Requemora (2002:259-260), le trait marquant de ce genre littéraire du XVII e siècle est celui "d'instruire et plaire ", à l'instar du binôme Merolla/Piccardo dont le premier rapporte des connaissances venues d'ailleurs tandis que le second les embellit, les enrobe d'une pâte littéraire. Mais les récits de voyage, devenus une littérature de masse dans la seconde moitié du XVII ${ }^{\mathrm{e}}$ siècle, n'en sont pas moins victimes de leur péché originel, celui de n'être par essence ni vrais ni faux, mais mi-réels, mi-fabuleux. La Breve e Succinta Relatione del Viaggio nel Regno di Congo n'échappe pas à cette règle et l'authenticité du texte est toute relative même si cet écrit constitue extrinsèquement un témoignage historique.

Comme tout récit du genre viatique, notre livre ne manque pas de décrire l'altérité. Son exotisme rassemble des topoï propres à ce genre, avec cependant des contingences de thèmes strictement liés à l'exercice d'un ministère religieux. Les typologies narratives ne cessent pas de s'entrecroiser et on trouve dans le texte, selon un ordre aléatoire ${ }^{5}$ :

- de la narration viatique: déplacements de l'auteur d'un lieu à un autre (de Naples à Lisbonne, de Lisbonne à São Salvador de Bahia, de là à sa mission du Soyo, etc.) ;

- des descriptions ethnographiques : sur les peuples indigènes que Merolla rencontra, mais aussi sur la faune et la flore nouvelles et exotiques ;

- de la chronique historique: concernant les successions dynastiques et les hauts-faits militaires au Royaume du Congo et dans les contrées alentours ;

- de la narration fantasmatique et providentialiste: dès lors qu'il s'agit d'une narration évoquant des références mythico-religieuses. De fait, nous trouvons dans le texte, nombre de renvois à une littéraire patristique ainsi qu'à des auteurs antiques tels que Virgile, Ovide ou Juvénal ;

- de la description épidictique : au sujet des prises de positions de Merolla sur des questions relatives à la traite transatlantique, au mercantilisme effréné et au comportement des acteurs coloniaux;

- des récits encomiastiques: il s'agit des passages portant sur des vies de prêtres missionnaires dont le zèle, la vie exemplaire voire le martyre frisent l'apologie ;

- de la narration morale : lorsque l'on est en présence de sermons appelant à la repentance, ou longues tirades contre de prétendus hérétiques, etc.

7 Notre attention, dans ce présent article, porte en revanche sur un point précis, au-delà des aspects mentionnés ci-dessus. Il y a dans Breve e Succinta Relatione, un certain nombre d'occurrences relatif à la couleur de peau des Africains que Merolla côtoya. 
Plusieurs passages du texte désignent le sujet Noir en fonction d'un colorisme ${ }^{6}$. En effet, le comportement et les mauvais travers des Congolais ou autres africains que relève le missionnaire capucin, s'expliqueraient par un essentialisme faisant que chaque action de ces derniers est conditionnée par leur couleur de peau, c'est-à-dire par leur noirceur.

\section{Loin du « bon sauvage »}

Le concept du «bon sauvage » n'était encore que peu présent, à la fin du XVII ${ }^{e}$ siècle, dans les débats européens. L'œuvre de Merolla n'était pas concernée par ces questions de relativisme de pensée qui sans doute auraient été, pour ce prêtre, des chimères voire des sottises de salon. C'est au siècle suivant qu'est posée l'épineuse question philosophique, à savoir la supériorité de l'état de nature par rapport à celui de culture. De plus, l'anticléricalisme des Lumières qui prit pied en Europe fut diamétralement opposé à l'interventionnisme et à la morale de l'église. À l'encontre du paradigme visant à mettre en avant les vertus d'un homme immaculé, écrivains et voyageurs ont cherché à promouvoir les bienfaits de l'existence simple et bucolique des peuples exotiques qui s'étaient maintenus dans un âge d'or, loin des vices des sociétés bourgeoises et industrielles ${ }^{7}$. Forts de ces principes utopiques, les intellectuels illuministes (Defoe, l'abbé Prévost, Montesquieu ou Rousseau, etc.) jugèrent la société européenne décadente, artificielle, corrompue par le luxe, et préconisèrent des réformes dans des domaines comme la justice et l'éducation ${ }^{8}$. Cependant, ces idées nouvelles se transformèrent parfois chez les défenseurs $\mathrm{du}$ « bon sauvage » en formules jusqu'au-boutistes :

Ce n'est pas au fond des forêts ; c'est au centre des sociétés policées qu'on apprend à mépriser l'homme et à s'en méfier. (Abbé Raynal, $1780: 266$ )

Si j'étais chef de quelqu'un des peuples de la Nigritie, je déclare que je ferais élever sur la frontière du pays une potence où je ferais pendre sans rémission le premier Européen qui oserait y pénétrer, et le premier citoyen qui tente d'en sortir. (Rousseau, 1819 : 124-125)

9 L'écriture de la Breve e Succinta Relatione, prisonnière d'un carcan moral, chargée de préjugés et d'idées préconçues, se situe aux antipodes de cet idéal qui tendait exagérément à décrire les sociétés dites primitives sous des traits affables et hospitaliers. Il en ressort, outre la pesanteur stylistique, une sémantique qui connote sans cesse l'autre en le dépeignant sous des traits péjoratifs et en proférant à son endroit des jugements nauséabonds. Néanmoins, ce sentiment de "suprématisme racialiste » n'était pas répréhensible au regard de l'époque, du milieu professionnel et du bagage idéologique de Merolla et des siens ${ }^{9}$; bien au contraire, l'invective et la stigmatisation étaient plutôt les marques d'un tempérament bien trempé, celui du pieux évangéliste attaché à convertir et à faire prospérer la mission.

C'est sans doute pour ce trait d'époque, qui catégorise plus qu'il n'analyse, que la deuxième partie du livre commence par une dissertation, du reste superfétatoire, sur l'origine de la couleur de peau des Noirs. Ne sachant à quel saint se vouer pour expliquer cette chimère, Merolla ou Piccardo semblent passer en revue les quelques auteurs ayant donné une opinion du phénomène mais tout reste du domaine mythicobiblique et non-scientifique.

Questa, la di cui negrura e corporal caligine delle sue foliginose nazioni, o dal clima

e vicinanza del Sole, o più tosto, all'assentire di molti, da stirpe, descendenza e 
prosapia il suo principio ottenne. Imperoché Siviglia, tenendo la distanza dal circolo Equinoziale verso Tramontana circa 38 gradi, produce li suoi Popoli bianchi ; vicino il fiume della Plata, oltra l'Equinozziale nell'istessa lontananza nascono gli uomini di color di castagna e ferrigno ; e presso il Capo di Buona Speranza, che quantunque tenghi più dell'Orientale, ha nulla di meno la medesima distanza verso l'Equinozziale, ed escono alla luce li Nazionali affatto negri, non dissomiglianti alla pece per il nereggiante colore. $(258)^{10}$

D’abord, ceci passe par Pline l'Ancien (23-79) : «En raison de la proximité du soleil, les habitants sont brûlés ». Puis, on trouve Girolamo Cardano (1501-1576) : "Ceux-ci semblent (être tels) plus en raison de leur race que de la nature de l'air » et Ovide (43 av. J.-C - 17) : " [...], le sang des Éthiopiens, attiré par la chaleur à la superficie de leur corps, leur donna cette couleur d'ébène qui depuis leur est devenue naturelle. ». Cette assertion vient des Métamorphoses et correspond à l'une des transformations du chaos primordial, celle de Phaéthôn, fils d'Hélios et de Clyméné. Phaéthôn, selon le mythe, pour son arrogance, perdit le contrôle de son char et pour cette mauvaise manœuvre, le continent africain se tarit, se transforma en désert et la peau des hommes se brûla et se fit noire. Cette histoire étant évidemment une jolie métaphore sur la nocivité des rayons du soleil ! En dernier lieu, on rencontre Isidore de Séville († 636) et Paolo Aresi (1574-1644) dont les opinions sont en phase avec celles que pouvaient avoir un missionnaire de XVII ${ }^{\mathrm{e}}$ siècle : "Il est absolument certain que l'origine de la noirceur n'est pas due à la région, en raison de l'ardeur du soleil, comme on a pu le croire, mais bien à la lignée de cette race, qui dérive du sang de Chus. » (José Sarzi Amade, op. cit., vol. 1, p. 171, notes 317-320).

\section{Un distinguo nécessaire a la prosperità de la mission}

12 L'enjeu singulier qui se posa pour Merolla et les siens fut celui de témoigner auprès de leur institution de Propaganda Fide et de leurs lecteurs du protagonisme dont ils firent preuve en mission. Afin de galvaniser les intérêts pour l'œuvre missionnaire, un subtil procédé se déploie dans la narration, consistant d'un côté à encenser les nouvelles ouailles et de l'autre à exécrer ceux qui refusent la religion. L'effet escompté est de poser toujours plus loin les jalons de l'évangélisation et de repousser les frontières du paganisme. Ainsi observe-t-on qu'une «diabolisation" s'effectue sur les sujets étrangers aux territoires christianisés ou qui sont des obstacles à l'avancée de la mission, tandis que ceux qui sont entrés ou qui entreraient dans le giron de la religion sont décrits avec mansuétude.

\section{Moutons dociles}

13 Certaines allusions de Merolla laissent entendre que, parmi les missions capucines qui avaient été mises en place au Royaume de Kongo dans un laps de temps assez rapide (1647-1654) ${ }^{11}$, celle du Soyo était la meilleure, faisant office de mission exemplaire et de bastion de la foi. Conscient de la faveur qui lui a été accordée par le préfet P. Paolo Francesco da Porto Maurizio (José Sarzi Amade, op. cit., vol. 1, p. 82, note 133), il écrit :

Quantunque io mi trovassi estenuato da gli strapazzi del viaggio, considerando nondimeno che il cammino era per mare, non volli perdere sì buona congiontura, tanto più ch'essendo la Missione di Sogno, una delle migliori e la più antica di quante n'abbiano, in consequenza è tra di noi la più stimata, e per le pianure non malagevoli al cammino, e per lo fiume, per cui, navigando, si può andar in molti 
luoghi, e sopra tutto per la pronta obedienza di quella gente, docile più d'ogn'altra.

$(72-73)^{12}$

14 Outre des conditions de vie relativement favorables par rapport aux autres établissements, c'est vraisemblablement la docilité des habitants de Soyo qui est remarquable et leur attitude à recevoir volontiers la religion chrétienne. Cette prédisposition morale n'est pas négligeable pour un missionnaire dont le devoir est de « ramener les brebis égarées au bercail », et possiblement en douceur, sans rencontrer de résistance. Merolla, au moment de sa venue dans ce comté, sait que près de deux siècles de christianisme en ces terres le contemplent et c'est pourquoi il aime à rappeler, quoique de façon très sommaire et partisane, l'aventure des Pères fondateurs au Royaume de Kongo, successivement des Dominicains puis les Franciscains de l'Observance. Ces deux ordres purent, d'après lui, semer en terrain fertile et, par leur labeur, faire fructifier comme il se doit la vigne du Seigneur. D'ailleurs, ce dernier en est reconnaissant car les Capucins sont arrivés, grâce à leurs prédécesseurs, dans un endroit où ils ont été accueillis avec aménité et en grande pompe. En effet, c'est bien en ces lieux que fut baptisé, avant même le roi de Kongo, le comte de Soyo, un jour de Pâques, le 3 avril 1491, et c'est là aussi que la première délégation capucine fut reçue avec la même ardeur au mois de mai-juin 1645 (José Sarzi Amade, op. cit., vol. 2, I. 4., p. 46 et sqq ; II. 2., p. 68 et sqq). Merolla tient alors à rappeler que sa mission est à la fois la plus ancienne, la plus prestigieuse et de loin la plus prolifique [pp. 76-80]. Les premières impressions de Capucin quand il arriva à Mpinda, ville portuaire fondée par les premiers colons portugais, furent positives ; il fut même stupéfié de voir autant de fidèles dans la plus vieille église du royaume, dédiée à la Vierge Marie [p. 87]. Il est impressionné par le respect intégral de la liturgie dans les églises des habitants du Soyo, leur grande ponctualité dans les offices et surtout par la chasteté et la bonne conduite mutuelles entre conjoints. Notre missionnaire dit à ce propos, non sans extrapoler, n'avoir jamais vu dans le comté aucun enfant illégitime ou métis comme on en trouve à foison ailleurs, dans les endroits non chrétiens. (232-233).

Le même constat est dressé à Kiova kia Nza, autre mission du comté de Soyo, où Merolla se félicita de la grande piété des gens qui venaient à sa rencontre, certaines mères pauvres affrontant jusqu'à cinq à six jours de marche pour y venir baptiser leurs nourrissons ou se confesser en couvrant les frais d'un interprète. Notre prêtre déclare même avoir administré ce sacrement à cinq cents personnes en une seule journée, zèle qu'il juge supérieur à celui déployé en Europe. Par contre, il déplore le peu de missionnaires présents sur place, ce qui est une entrave à la pérennisation de la religion en ces lieux (134-135). Ce constat est diffus dans la Breve e Succinta Relatione : c'est justement ce manque d'évangélistes qui, selon Merolla, rendit la tâche si pénible et fut la cause d'échecs dans les missions et de la perte de milliers d'âme dans des contrées, certes, rétives au message de la foi mais où le temps aurait fait son œuvre, pensait-il ${ }^{13}$ :

Se vi permanessero Sacerdoti a sufficienza, singolarmente dell'inviati dalla Sacra Congregazione e S. Sede Apostolica, direi non esser difficile la Conversione di tutti quei popoli dell'Etiopia inferiore. (410)

[...] per mezo de' sacri ammaestramenti, la verità Cattolica, volentiermente si piegano, disviticchiati dall'ostinazione pestifera in cui radicalmente viziati vivevano; [...]. (427) $)^{14}$

16 Bien conscient que la tâche de sauver les âmes pouvait être améliorée, Merolla prend congé de l'Afrique à cause de la maladie qui l'opprime. Pourtant, il ne renonce pas à 
persévérer dans cette voie car nous savons qu'il fut très rapidement de retour en mission, dès 1693, certainement avec l'idée claire d'achever le travail laissé derrière lui au Royaume de Kakongo (vol. 2, Introduction, 5. 3 et 5. 4) ${ }^{15}$. Nous pouvons toutefois entrevoir chez lui, dans les toutes dernières pages de son texte, un sentiment mêlé de nostalgie et de compassion quand il déplore de ne pas avoir été au bout du projet de conversion au Kakongo ("quei poveri Regni bisognosi di scorta per la strada del Cielo ») ${ }^{16}$ et quand il nomme les Africains « denigrati Popoli » (peuples dénigrés), comme s'il voulait résumer ainsi toute la mauvaise réputation dont ces derniers pâtissent (429-430).

\section{Brebis galeuses}

17 Outre les invariables motifs de réprobation adressés aux Noirs, Merolla signale aussi les imperfections de Blancs implantés en Afrique. C'est le cas du Royaume de Benguela (Angola) qui fut pendant un certain temps évangélisé. Il y déplore la mauvaise conduite des colons blancs, insidieuse pour la progression missionnaire, car les habitants suivirent le mauvais exemple de ces colons. Les vindictes contre les prêtres s'y multiplièrent de sorte que le christianisme recula et disparut (60-61). De plus, dans ce même royaume, le Capucin semble dénoncer un autre travers de ces «Blancs des tropiques" (Créoles). En effet, ceux-ci profitent des mœurs légères des Noirs qui, pratiquant une sorte de drôle de maquerellage, capturent ceux qui sont surpris dans des actions licencieuses et les emprisonnent. Les colons, complices, rachètent les prisonniers pour les destiner à l'esclavage (67-69).

18 C'est à Luanda que Merolla a le plus scruté le mode de vie des habitants. Là encore, il va de critiques en critiques face à un tableau peu orthodoxe à ses yeux. Il reproche aux femmes blanches de ces lieux d'avoir été mal éduquées, influencées par leurs nourrices noires, c'est pourquoi elles ont les mêmes comportements. Il constate qu'elles sont très autoritaires et désobligeantes envers leurs maris au point de leur jouer toutes sortes de vilains tours. De même, ce sont elles qui tiennent les rênes du pouvoir au sein des familles et qui ont subverti le système des biens successoraux, de manière à ce que seule la gent féminine les obtienne de génération en génération. Merolla est également gêné de leur pompe qui varie selon les circonstances et de leur légèreté quant à la fréquentation des offices religieux, mauvais exemple une fois de plus pour les catéchumènes (375-379).

Bien sûr, la Relatione abonde en stigmatisations contre les Africains, et en cela ne peuvent manquer les poncifs sur ces derniers. Merolla les accuse d'être paresseux (ils pratiquent très peu la pêche [p. 86]), avides d'eau de vie ou de tabac (parfois utilisés comme appâts pour les faire passer aux aveux [p. 90]). Ils sont aussi taxés de "perfides» car souvent prompts à la désobéissance et à la calomnie envers les missionnaires (114-115), de "pervers» (414) parce qu'insensibles à la souffrance d'autrui et de « rusés » quand il est question de trouver les arrangements nécessaires à leur profit (416-418).

20 Si la mission de Soyo et ses fidèles a été souvent décrite par Merolla avec égards et sous des traits favorables, il emploie une prose distinctive pour désigner tout ce qui se situe hors du christianisme, dans un état d'infériorité marquée, comme pour séparer le bon grain de l'ivraie. 


\section{Le blanc c'est Blanc, le noir c'est Noir}

21 Il y a dans la Breve e Succinta Relatione, pour ceux que Merolla considère comme "Gentils ", c'est-à-dire impies et donc non chrétiens, une façon toute singulière d'assombrir leur portrait, presque à la manière d'un peintre qui manierait des nuances de clair-obscur sur ses toiles. En effet, c'est de façon binaire qu'il oppose la noirceur des incroyants à la blancheur des chrétiens et vice versa. Par colorisation symbolique, notre Capucin s'applique à connoter le champ du bien par le blanc et son contraire par le noir.

Ce procédé se systématise dans l'œuvre entière. On en trouve d'emblée l'empreinte dans le sonnet composé par Giuseppe de Magistris en l'honneur du ministère de Merolla dont la foi exemplaire a réduit les monstres noirs en cygnes joyeux, changé les corbeaux en cygnes et transformé les taupes aveugles en Argos [11v]. Le même champ lexical du clair-obscur apparaît chez Piccardo et s'exprime dans le Bréviloque (en introduction au livre) avec le dédain de circonstance :

Dall'oscurezze de' nericanti Torchi esce alla luce il Viaggio del P. Girolamo da Sorrento Sacerdote Cappuccino in un nero ed affumigato Mondo, dico ne' popoli Etiopeni dell'Africa Meridionale. [6v $]^{17}$

Dans l'Exastichon final (poème de six vers) dédié aux Capucins ayant traversé récemment l'Afrique pour des Missions, Piccardo les invite à purifier ces nations de leurs scories, de façon à ce que le noir corbeau devienne pure colombe et que les cœurs enduits de poix se fassent blancs comme neige (442). D'autres lourdes allusions aux corbeaux et aux colombes sont faites quand il s'agit d'illustrer ce qu'est un Noir qui prétendrait épouser une Blanche (404). Autre exemple: Merolla se réfère à une corneille croassante pour désigner une femme noire qui l'agaçait (352).

Dès les premières pages du livre Merolla dresse un bien sombre tableau de ses probables futurs catéchumènes. Le constat est sans appel et il semble que seul Dieu tout-puissant puisse délivrer ces peuples de l'emprise du mal. Là encore tout s'articule autour d'oppositions entre ombre et de lumière :

E perché il predicare ed evangelizzare la parola Divina a quei che sepolti nelle tenebre della propria ignoranza vivono, e siedono nell'ombre della morte, è uno de' più alti, degni e sublimi esercizii che si possa esercitare nella Chiesa militante ; [...], o pure con faticosi Esercizii di predicare a quelle barbare Nazioni, soffrire un lungo e continuato martirio, a fine di ridurle alla luce dell'Evangelo, alla cognizione del vero Dio ed alla verità della Fede, di cui affatto son prive ; [...]. (2-3) 18

Il use d'une expression du même acabit pour désigner le baptême: par exemple, baptiser tout un peuple noir, se traduira par le «blanchir avec l'eau de la source sacrée » (78). On trouve foison de qualificatifs qui emphatisent la couleur des Noirs, en faisant presque aux yeux du missionnaire, la cause de tous leurs maux (ignorance, ruse, etc.). Il les désigne tour à tour, en référence à leur peau, comme ayant le visage patiné de suie [p. 405], hommes noirs comme de la poix [p. 258] ou provenant d'un monde fumeux [p. 255]. Bref, le prêtre, les nommant de la sorte, décrit un endroit qui s'apparente, dans son imaginaire, à l'antre de l'enfer !

Quand les raisonnements racistes et abjects prennent le dessus, ils se manifestent dans de troublants raccourcis de la pensée. Merolla dira par exemple que les Noirs, ayant été ainsi peints par la Nature, ont à leur disposition toute une palette de sombres couleurs pour obscurcir l'esprit humain (418). Aussi, le missionnaire se plaint-il, au Soyo, de ne pas pouvoir converser avec plus de Blancs, une activité qui lui manque, dit-il au milieu 
de "gl'oscuramenti e negrezze di tanti negri ed oscuri Etiopi $»^{19}$ (217). De même, dans la prose de Merolla, le Blanc, par sa couleur, est toujours mis en avant comme débonnaire, dès lors qu'il sert de contrepoids pour souligner les actions répréhensibles du Noir.

La candeur, synonyme de blancheur immaculée et d'extrême pureté de l'âme, est une caractéristique du Blanc et indique une conduite vers laquelle doit tendre le Noir qui, lui, est mauvais et traitre par nature. Ici, parce qu'un enfant noir est blanc de cœur, il a eu plus tard accès au sacerdoce :

Erasi nel nostr'Ospizio fin da teneri anni allevato un figliuolo nativo del Contado, e per essere quanto nero nel corpo tanto più bianco e candido di cuore, [...] i nostri Frati lo ferono ordinar Sacerdote [...]. (268) 20

De ce fait, le Blanc qui possède la candeur se doit de montrer le bon exemple au Noir, qui est privé de cette qualité :

Se la legge di Dio non s'osserva candidamente da' Bianchi, come potrà aver osservanza con candidezza da' Neri ? (61) ${ }^{21}$

Les actions qui sont à l'initiative des Noirs doivent être considérées par les Blancs avec méfiance, à l'aune de leurs défauts intrinsèques :

L'Illustrissimo Prelato, nella di cui candidissima mente stavano sempre chiari e noti

li portamenti de' Neri, [...]. (210)

ed io non fidandomi troppo delle sue parole, [...] per esser lui oscuro e negro, [...].

$(335)^{22}$

Pour Merolla, le mélange des couleurs blanche et noire entre individus est exsudation impropre ( $"$ mescolamento feccioso dell'una e dell'altra Nazione $»^{23}$ (379)). Il peine tant à concevoir l'idée du métissage qu'il livre un tableau à la fois farfelu et horrifique de ce que sont les fruits d'une union entre personnes à la couleur de peau différente. Selon lui, ces procréations peuvent être monstrueuses :

altri figliuolini [...] in questa fosca e negra Etiopia nati : che sono taluno uscito dal seno materno colla barba e tutti li denti; forse (io direi) per dimostrare esser venuto in quel cieco ed affumicato Mondo, canuto per le maledicenze, ed invecchiato per i vizii ; così anco un bianco ed un negro in un medesimo parto prodotti alla luce ; e da Donna negra un altro bambino totalmente bianco partorito. $(254-255)^{24}$

30 Face au dénigrement des mariages mixtes, une incartade pouvait être tolérée à partir du moment où il s'agissait de servir les intérêts de la mission et de faire progresser l'évangélisation. En effet, Merolla relate le mariage d'une dignitaire blanche de São Tomé avec un souverain noir du Royaume d'Owerri (Bénin), lequel, grâce à cette union, se convertit au christianisme. Épouser le roi noir est une acceptation de martyre pour la cause de l'Église et de l'État. Mais en définitive, tant les missionnaires que les Portugais voyaient dans cet accord, une possibilité d'expansion (405-407).

31 L'écriture de ce bref article met en lumière les apriorismes raciaux qui transparaissent dans le récit de voyage Breve e Succinta Relatione del Viaggio nel Regno di Congo du Capucin italien, Girolamo Merolla. Par ailleurs, ceux-ci nous renseignent davantage sur la teneur culturocentriste de cet ouvrage. Au-delà de ces tournures langagières pouvant heurter nos sensibilités présentes, il faut rappeler que ce livre de la fin du XVII siècle se situe encore à la lisière du monde moderne et se voit alourdi par la rhétorique d'un missionnaire zélé qui désignait l'Africain, c'est-à-dire l'homme noir, par une taxonomie rude, propre à l'Ancien Régime.

Si nous avons trouvé la peine de souligner ce modus operandi qui met en exergue des problèmes inhérents à la rencontre, la découverte ou la considération de l'autre, on doit 
néanmoins faire grâce à Merolla de deux aspects touchant d'autres thèmes. D'une part, d'avoir souligné dans son texte et combattu, à son échelle, l'infamie du commerce triangulaire entre nations belligérantes (Portugal, Angleterre et Pays-Bas). D'autre part, il sut apporter sa pierre à l'édifice en ce qui concerne l'extension des connaissances ethnographiques sur l'Afrique subsaharienne, car à son époque, très peu de choses à ce sujet avaient été explorées et étaient connues du public européen. La divulgation du savoir sur les contrées exotiques commença, grâce aux encyclopédistes qui lisaient ce genre de littéraire, à partir de la moitié du XVIII ${ }^{e}$ siècle.

\section{BIBLIOGRAPHIE}

BALCAZAR, Fabricio E., Race, Culture and Disability: Rehabilitation Science and Practice, Jones \& Bartlett Learning Publishers, Boston, 2010.

CRO, Stelio, El buen salvaje y la edad moderna : Hackluyt, Montaigne y Pedro Mártir, in AIH. Actas X, Centro Virtual Cervantes, 1989, pp. 398-405.

FERRONE, Vincenzo, “Il problema dei selvaggi nell'Illuminismo italiano”, Studi Storici, Fondazione Istituto Gramsci, Anno 27, No 1, 1986, pp. 149-171.

MEROLla da Sorrento, Girolamo (P.), Breve e Succinta Relatione del Viaggio nel Regno di Congo nell'Africa Meridionale, fatto dal P. Girolamo Merolla da Sorrento, Sacerdote Cappuccino, Missionario Apostolico. Continente variati clima, arie, animali, fiumi, frutti, vestimenti con propie figure, diversità di costumi, e di viveri per l'uso humano. Scritto, e ridotto al presente stile Istorico, e narrativo dal P. Angelo Piccardo da Napoli Predicatore dell'istess'Ordine. Diviso in due parti. Dedicato all'Emin.mo e Rev.mo Cardinal Acciaioli, Per Francesco Mollo, Napoli, 1692.

RAYNAL, Guillaume-Thomas-François (abbé), Histoire philosophique et politique des établissemens et du commerce des Européens dans les deux Indes, Volume 4, Genève, chez J. L. Pellet, 1780.

REQUEMORA, Sylvie, «L'espace dans la littérature de voyages », Études littéraires, vol. 34, n 1-2, 2002, pp. 249-276.

RousSEAU, Jean-Jacques, Discours, Chez Madame veuve Perronneau, Paris, 1819.

SARZI AMADE, José, (tapuscrit soumis pour publication, janv. 2017). Édition critique de la Breve e Succinta Relatione del Viaggio nel Regno di Congo [...] (1692) de Girolamo Merolla da Sorrento, vol. 1, $310 \mathrm{p}$.

SARZI AMADE, José, (tapuscrit soumis pour publication, janv. 2017). Reédition, contextualisation et analyse du récit de Girolamo Merolla da Sorrento, missionnaire capucin au Royaume de Kongo de la fin du XVII siècle, vol. 2, 408 p.

TURCHETTA, Barbara, Missio antiqua : padre Giacinto da Vetralla missionario in Angola e in Congo : un cappuccino italiano del secolo XVI tra linguistica e antropologia, Viterbo, Sette città, 2007. 


\section{NOTES}

1. Titre complet: Breve e Succinta Relatione del Viaggio nel Regno di Congo nell'Africa Meridionale, fatto dal P. Girolamo Merolla da Sorrento, Sacerdote Cappuccino, Missionario Apostolico. Continente variati clima, arie, animali, fiumi, frutti, vestimenti con propie figure, diversità di costumi, e di viveri per l'uso humano. Scritto, e ridotto al presente stile Istorico, e narrativo dal P. Angelo Piccardo da Napoli Predicatore dell'istess'Ordine. Diviso in due parti. Dedicato all'Emin.mo e Rev.mo Cardinal Acciaioli. In Napoli, Per Francesco Mollo, 1692.

NB : la numérotation insérée entre crochets dans le présent article renvoie à ce texte.

2. Notre étude (Sarzi Amade, 2017) comprend deux volumes (tapuscrits soumis pour publication en janvier 2017) intitulés : Édition critique de la Breve e Succinta Relatione del Viaggio nel Regno di Congo [...] (1692) de Girolamo Merolla da Sorrento - vol.1, 310 p. - et Réédition, contextualisation et analyse du récit de Girolamo Merolla da Sorrento, missionnaire capucin au Royaume de Kongo de la fin du XVIIe siècle. - vol. 2, 408 p. - Pour tout approfondissement, s'y référer.

3. Il était l'amanuensis, l'écrivain-secrétaire à qui l'auteur [Merolla] dicta.

4. $\mathrm{TdA}$ : «Bien que je puisse même ajouter que cette brève, et succincte Relation était encore le fruit de la brièveté du temps, motivée par la sollicitude de l'Auteur à un second départ pour ces marécages africains, [...]. »

5. L'ensemble de ces typologies narratives a été longuement traité ; cf. José Sarzi Amade, op. cit., vol. 2.

6. Le colorisme consiste à marquer une différence entre les individus à la peau claire et ceux, a contrario, à la peau sombre. Les sujets plus foncés sont considérés suivant ce critère, comme moins aptes ou moins nobles. La couleur de peau est ici un discriminant comme peuvent l'être d'autres éléments appartenant au phénotype (Fabricio E. Balcazar, 2010 : p. 82).

7. Pietro Martire d'Anghiera (1457-1526) dans son œuvre De Orbe Novo avait, peu de temps après la découverte des Amériques, démontré une grande empathie pour ces peuples du Nouveau Monde. Il les pensait vivant dans un âge d'or, alors que les Européens l'avaient quitté depuis longtemps et appartenaient eux à l'âge de fer à cause de leur ruse. Il les voyait encore plus heureux que les Italiques que Virgile fait rencontrer à Énée. Sorte d'hommes nouveaux, les indigènes d'Amérique, pensait-il, méconnaissaient le « toi » et le « moi », source de tous les maux (Stelio Cro, 1989 : 398-400).

8. Pour une connaissance plus précise de la diffusion du mythe du «bon sauvage » (cf. Vincenzo Ferrone, 1986 : 149-171).

9. Il est fort probable que les Capucins aient adhéré aux statuts de pureté de sang en vigueur dans les territoires espagnols et portugais. Par cette législation, une nette différente était faite entre les vieux et les nouveaux chrétiens et la dépréciation des non catholiques était très marquée.

10. TdA : "Cette noirceur et brume corporelle des nations fuligineuses tire son principe, ou du climat et proximité du Soleil, ou plutôt, avec l'acceptation de beaucoup, de la souche, descendance et lignée. Étant donné que Séville se situe à une distance de 38 degrés du cercle équinoxial en direction de la Tramontane, elle produit jusque-là les Peuples Blancs ; près du Rio de la Plata, dépassant la ligne équinoxiale à la même distance, naissent les hommes de couleur marron et ferreuse, et au Cap de Bonne-Espérance, même s'il appartient davantage à l'Orient, il n'a rien de moins que la même distance vers la ligne équinoxiale, apparaissent les habitants complètement noirs, semblables à la poix en raison de leur noirâtre couleur. "

11. Entre 1648 et 1654, les Capucins avaient fondé dans les chefs-lieux des différentes provinces de Kongo, des missions à: Mbanza Kongo, Mbanza Soyo, Mbanza Mpemba et Mbanza Nsevo. Quelques autres missions secondaires se trouvaient dans les comtés de Soyo (Kiova kia Nza et Matari), Nsundi, Mbata, Mpemba, Mbamba et dans les territoires des alentours comme Wandu et Nkusu (Barbara Turchetta, 2007 : 41). 
12. TdA : «Bien que je me trouvasse exténué par les fatigues du voyage, considérant malgré tout que le chemin était par mer, je ne voulus perdre une aussi bonne conjoncture, d'autant plus que la Mission de Soyo, une des meilleures et la plus ancienne de celles que l'on a, par conséquent elle est parmi nous la plus estimée, et pour les plaines non malaisées à la marche, et pour le fleuve, le long duquel, en naviguant, on peut aller en de nombreux endroits, et surtout pour la prompte obéissance de ce peuple, docile plus que tout autre. »

13. Cet aveu d'impuissance chez notre prêtre, avait trouvé son explication dans les requêtes qu'il adressa à la Congrégation De Propaganda Fide, précisément pour accorder l'envoi de nouveaux missionnaires au royaume de Kakongo avant même son retour pour l'Italie (cf. José Sarzi Amade, op. cit., vol. 2 : pp. 289-293, annexe 1, lettres 1-3).

14. TdA : "S'il y demeurait suffisamment de prêtres, en particulier ceux envoyés par la Congrégation et le Saint-Siège Apostolique, je dirais qu'elle ne serait pas difficile la Conversion de tous ces peuples de l'Éthiopie inférieure.»

«[...] par voie des saintes instructions, volontiers ils se plient à la vérité Catholique, purifiés de leur obstination pestiférée dans laquelle ils vivent corrompus ; [...]. »

15. Selon Merolla ce royaume en voie de christianisation pouvait être gagné à l'Évangile. On peut lire toute la détermination de notre missionnaire dans ses lettres à la Congrégation De Propaganda Fide: "[...] per la grande necessità d'operari che si ricerca in quelli regni. [...] sarebbe facilissimo ridurlo alla santa fede con tutto il regno, [...]. », cf. José Sarzi Amade, op.cit., vol. 2, annexe 1 : SC Africa, Angola, Congo, vol. 2, fo 529[r] (lettre 1), fo 571[r/v] (lettre 3).

16. $\mathrm{TdA}:$ « ces pauvres Règnes ayant besoin d'escorte pour le chemin du Ciel. »

17. TdA : «Des ténèbres des noirâtres Pressoirs est sorti le Voyage du P. Girolamo de Sorrente Prêtre Capucin, dans un Monde noir et fumant, je dis parmi les peuples Éthiopiques de l'Afrique Méridionale. »

18. TdA : "C'est parce qu'évangéliser et prêcher la parole divine à ceux-là, qui enfouis dans les ténèbres de leur ignorance, vivent, et prennent place dans l'ombre de la mort, qu'il est un des plus nobles, dignes, et sublimes exercices, que l'on puisse exercer dans l'Église militante ; [...], ou bien avec de laborieux exercices, de longues souffrances, et de constant martyre, pour prêcher à ces Nations barbares, jusqu'à les réduire à la lumière de l'Évangile à la connaissance du Dieux vrai, et à la vérité de la Foi, dont elles sont totalement dépourvues ; [...]. »

19. $\mathrm{TdA}:$ « les assombrissements et noirceurs de tant de nègres et obscurs Éthiopiens. »

20. TdA : «Il y avait un enfant natif du Comté élevé dans notre Hospice depuis son jeune âge, et pour autant qu'il eût le corps noir, plus blanc et candide de cœur il était, [...] nos Frères le firent ordonner Prêtre [...]. »

21. TdA : «Si la loi de Dieu, n'est pas observée candidement par les Blancs, comment pourrait-elle avoir avec candeur l'observance avec Noirs?»

22. TdA : «Le Très Illustre Prélat, dont l'esprit très pure comprenait et connaissait la conduite des Noirs, [...]. »

« et moi ne me fiant pas trop à ses paroles [d'un rival du roi], parce qu'il était obscur et noir, [...].»

23. TdA : « désagréable mélange de l'une et de l'autre Nation »

24. TdA : «D'autres petits enfants [...] dans cette sombre et noire Éthiopie sont nés : un, sorti du ventre maternel avec de la barbe et toutes les dents; sans doute (dirais-je) être venu dans ce Monde aveugle et fumant, gris pour les calomnies et vieilli par les vices; au même titre qu'un blanc et un noir produits d'une même naissance ; et un autre enfant né entièrement blanc d'une Femme noire. » 


\section{RÉSUMÉS}

Cet article traite certains motifs du récit de voyage d'un Capucin missionnaire du nom de Girolamo Merolla. Son texte donne lieu à des épisodes mettant en scène la rencontre entre deux cultures diverses et moralement antagonistes. Parfois, elle suscita troubles, incompréhensions et réactions culturocentristes. En effet, dans le récit de la Breve e Succinta Relatione (1692), il est souvent question d'une rhétorique dépréciative empreinte de « colorisme » envers les Africains.

This article analyses some patterns within the account of the voyage to Congo made by the Capuchin missionary Girolamo Merolla. His text gives rise to a series of episodes which shed light on the encounter between two different and morally antagonistic cultures. Sometimes, this coming upon face-to-face will generate troubles, incomprehension and culture-centrist reactions. As a matter of fact, it is common to find, within the narrative of Breve e Succinta Relatione (1692), a depreciative rhetoric, imprinted with "colorism" toward Africans.

INDEX

Mots-clés : Merolla, Congo, mission, Noirs, colorisme

مغولا, ألكونغو, السود, مهمة, لون العرقفهرس الكلمات المفتاحية:

Keywords : Merolla, Congo, mission, Blacks, colorism

\section{AUTEUR}

JOSÉ SARZI AMADE

Université Aix-Marseille 